\title{
Reflexiones, metodología, propuestas y experiencias sobre información y documentación del patrimonio documental en la universidad
}

\author{
María Teresa Munera Torres \\ Universidad de Antioquia (Colombia)
}

\section{Resumen}

Reflexión sobre lo que es el patrimonio documental, con base en la definición que se le brinda en diferentes ámbitos y en especial la que aporta la UNESCO. Seguidamente se establece el nexo entre lo que significa la universidad como institución de educación superior y su relación con el patrimonio, ya que es una entidad que produce una gran cantidad de conocimiento para el beneficio y provecho de la sociedad y un abundante acopio de todo su producto intelectual, científico, cultural, administrativo y artístico desde el momento de su origen hasta la actualidad, el cual se convierte en evidencia de su función y proyección a la comunidad. Esta relación se ilustra mediante la presentación del proyecto de investigación titulado "Patrimonio documental Universidad de Antioquia, 200 años". Al final se presentan unas conclusiones mediante las cuales se logra establecer lo que significa el patrimonio documental en la universidad y, de manera específica, en la Universidad de Antioquia.

Palabras clave: Patrimonio cultural. Patrimonio documental. Universidad.

\section{Abstract}

Critical reflections on the concept of documentary heritage, based on some of its different definitions, specially the one from UNESCO. The link between the university, as a higher education institution, and heritage management is explored: the university is an institution that creates and preserves a huge amount of knowledge for the benefit of the rest of the society. This link is exemplified by the project "Documentary heritage, University of Antioquia, 200 years". The conclusions establish the meaning of documentary heritage in the University and, specifically, in the University of Antioquia.

Keywords: Cultural heritage. Documentary heritage. University. 


\section{Introducción}

Al concebir el patrimonio, se puede traer a efecto la definición otorgada por el diccionario de la lengua española: "Hacienda que alguien ha heredado de sus ascendientes"; "Conjunto de los bienes propios adquiridos por cualquier título" (Real Academia Española, 2001, p. 1703). Dentro de dicho patrimonio, se destaca lo que es el patrimonio cultural, como legado que hereda un pueblo o región y que está conformado a su vez por todas las manifestaciones humanas, materiales, intelectuales y artísticas que den cuenta de su identidad. El legado de conocimiento es una de las riquezas que mayor influencia tienen en el desarrollo y sustento de una institución, de una comunidad, de un país, de una región y de toda la humanidad en general. Con el transcurrir del tiempo se va conformando un conglomerado de aportes que van pasando de generación en generación y a su vez van marcando la pauta para el origen de los procesos de cambio y crecimiento de las diferentes organizaciones, culturas y sociedades. Para el caso específico de las universidades, el conocimiento que se produce continuamente en sus diferentes espacios - académicos, administrativos, investigativos, lúdicos y de proyección - se constituye en el gran baluarte que les servirá de apoyo para la construcción de las nuevas dimensiones de universidad que requiere la sociedad a la que se proyecte, en cada momento de su devenir histórico. Por tanto, es necesario vincular este legado de conocimiento con el patrimonio, como manifestación que debe ser valorada y conservada para beneficio no solo de la universidad como tal, sino como el cimiento del desarrollo histórico, científico y social de la comunidad en general.

Para tener una idea de lo que significa el conocimiento generado en la universidad como patrimonio, se darán a conocer a continuación algunas ideas sobre la definición de patrimonio en términos generales; y, dentro del mismo, se precisará lo que es el patrimonio natural y cultural y, a su vez, dentro de este último se definirán los patrimonios culturales tangible e intangible. Además, se enfatizará en lo que es el patrimonio documental, y en especial el de una entidad de educación superior. Luego se describirán los pasos a seguir en una propuesta de conformación del patrimonio documental de una universidad y, por último, se presentará un recuento de cómo se está llevando a cabo dicho trabajo académico en la Universidad de Antioquia.

\section{2. ¿Qué se entiende por patrimonio?}

$\mathrm{Al}$ abordar el término patrimonio, se encuentra que proviene de la expresión latina patrimonium y su significado se le atribuye solo a aquellos objetos que presentan un valor histórico y una relación directa con el pasado. No obstante, algunos estudiosos del tema le han sumado a la anterior definición el valor y aporte cultural que pueden brindar manifestaciones artísticas intangibles, tales como 
la música, las fiestas folclóricas o tradicionales de determinada región, mediante las cuales se da a conocer la identidad de una comunidad específica. De ahí, que entidades como la UNESCO, etc., lo definan como (República de Chile, s. f.)

el conjunto de bienes valiosos, materiales o inmateriales, heredados de los antepasados. Ellos reflejan el espíritu de una época, de una comunidad, de una nación, y de la propia humanidad. El patrimonio que se va decantando de generación en generación conforma el sello distintivo de un pueblo. Por ello el patrimonio es una manera de acercarse al conocimiento de la identidad nacional.

Al continuar indagando sobre lo que se entiende por patrimonio, se pueden lograr varios significados de esta expresión. Por ejemplo, el diccionario de la lengua española lo define como "Hacienda que alguien ha heredado de sus ascendientes", o como el "Conjunto de los bienes propios adquiridos por cualquier título" (Real Academia Española, 2001, p. 1703). Otros entienden el patrimonio como aquellos bienes muebles e inmuebles de valor cultural que pertenecen a una comunidad y que, por lo tanto, merecen un régimen especial de atención, cuidado y protección. También se incluyen en el significado de patrimonio los bienes tangibles e intangibles heredados de los antepasados; el ambiente donde se vive; los campos, ciudades y pueblos; las tradiciones y creencias que se comparten; los valores y religiosidad; la forma de ver el mundo y adaptarse a él.

La UNESCO también enfoca la definición dentro de la expresión de patrimonio mundial, compuesto fundamentalmente por dos tipos de patrimonio: el cultural y el natural. Sin embargo, algunos como el Ministerio de Educación de Chile, clasifican al patrimonio desde diferentes puntos de vista. Así (República de Chile, s. f.), dentro del patrimonio general, conciben dos tipos fundamentales: el patrimonio natural y el patrimonio documental.

El patrimonio natural "se manifiesta en los monumentos constituidos por formaciones físicas y biológicas o por grupos de esas formaciones que tengan un valor universal excepcional desde el punto de vista estético o científico. Las formaciones geológicas y fisiográficas y las zonas estrictamente delimitadas que constituyan el hábitat de especies animal y vegetal amenazadas, que tengan un valor universal excepcional desde el punto de vista estético o científico, los lugares naturales o las zonas naturales estrictamente delimitadas, que tengan un valor universal excepcional desde el punto de vista de la ciencia, de la conservación o de la belleza natural" (UNESCO, 1972). La UNESCO lo define como "aquellos monumentos naturales, formaciones geológicas, lugares y paisajes naturales, que tienen un valor relevante desde el punto de vista estético, científico y/o medioambiental. El patrimonio natural lo constituyen las reservas de la biosfera, los monumentos naturales, las reservas y parques nacionales, y los santuarios de la naturaleza" (UNESCO, 1972). 
El patrimonio cultural se presenta fundamentalmente en los bienes culturales que la historia le ha legado a una nación y por aquellos que en el presente se crean y a los que la sociedad les otorga una especial importancia histórica, científica, simbólica o estética. Se constituye, por tanto, en el legado recibido de los antepasados y que viene a ser el testimonio de su existencia, de su visión de mundo, de sus formas de vida y de su manera de ser, y es también la herencia que se deja a las generaciones futuras.

Para la UNESCO, el patrimonio cultural vendría a designar monumentos, grupos de edificios y sitios que tienen valor histórico, estético, arqueológico, científico, etnológico o antropológico. En el patrimonio cultural por lo tanto tienen cabida (UNESCO, 1972)

Los monumentos: obras arquitectónicas, de escultura o de pintura monumentales, elementos o estructuras de carácter arqueológico, inscripciones, cavernas y grupos de elementos, que tengan un valor universal excepcional desde el punto de vista de la historia, del arte o de la ciencia; los conjuntos: grupos de construcciones, aisladas o reunidas, cuya arquitectura, unidad e integración en el paisaje les dé un valor universal excepcional desde el punto de vista de la historia, del arte o de la ciencia; los lugares: obras del hombre u obras conjuntas del hombre y la naturaleza así como las zonas incluidos los lugares arqueológicos que tengan un valor universal excepcional desde el punto de vista histórico, estético, etnológico o antropológico.

Se podría analizar cómo, dentro del mismo patrimonio cultural, se pueden determinar ciertos tipos específicos.

Así, el patrimonio cultural urbanístico es definido como aquel que "abarca todos aquellos elementos arquitectónicos, urbanos y de paisaje que tienen un significado especial para las personas. Su importancia radica en el valor histórico, simbólico o vivencial, en las cualidades ambientales, arquitectónicas o porque representan la forma de habitar de una comunidad" (Gobernación de Antioquia, 1996, p. 5).

El patrimonio cultural tangible se define como la expresión de las culturas mediante grandes realizaciones materiales. A su vez, el patrimonio tangible se puede clasificar en mueble e inmueble. El patrimonio cultural tangible mueble es aquel tipo de patrimonio que comprende los objetos arqueológicos, históricos, artísticos, etnográficos, tecnológicos, religiosos y aquellos de origen artesanal o folclórico que constituyen colecciones importantes para las ciencias, la historia del arte y la conservación de la diversidad cultural de un determinado país. Entre dichos objetos se pueden identificar las obras de arte, los libros manuscritos, los documentos, los artefactos históricos, las grabaciones, las fotografías, los documentos audiovisuales, las artesanías y otros objetos de naturaleza arqueológica, histórica, científica y artística. Por su parte, el patrimonio cultural tangible inmueble tiene que ver más con lugares específicos, edificaciones, obras de 
ingeniería, centros industriales, conjuntos arquitectónicos, zonas típicas y monumentos de interés o valor significativo desde diferentes puntos de vista, como el histórico, el arquitectónico, el arqueológico, el artístico, el científico, entre otros. Estos bienes culturales inmuebles son obras o producciones humanas que no pueden ser trasladadas de un lugar a otro, ya sea porque son estructuras (por ejemplo, un edificio), o porque están en inseparable relación con el terreno (por ejemplo, un sitio arqueológico).

El patrimonio cultural intangible está conformado por las expresiones culturales por excelencia. Se destacan aquí los legados de saber fundamentados en la tradición oral. Se podrían enmarcar dentro del patrimonio intangible todas aquellas manifestaciones espirituales, intelectuales, afectivas que le dan la identidad a una determinada sociedad, tales como costumbres, sistemas de valores, religión y creencias, leyendas, literatura y en general todo aquello que forma parte de su identidad cultural.

Finalmente, luego de mirar lo referente al patrimonio y a sus dos principales vertientes, el patrimonio natural y el patrimonio documental, se puede deducir claramente que el patrimonio documental estaría enmarcado dentro del patrimonio cultural tangible mueble. Por lo tanto, este tipo de patrimonio estaría conformado por "bienes reunidos o no en archivos y bibliotecas, que conforman un patrimonio documental bibliográfico" (Gobernación de Antioquía, 1996, p. 5). Resulta interesante reflexionar sobre cómo el concepto de documento abarca todas las manifestaciones del lenguaje natural o artificial, en diferentes formatos de carácter impreso, gráfico, sonoro, electrónico, digital, entre otros. Por tanto, formarían parte del patrimonio documental (Hernández, 2002, p. 201)

Aquellos documentos generados, conservados o reunidos, en el ejercicio de su función, por cualquier organismo o entidad de carácter público, por las personas jurídicas en cuyo capital participe mayoritariamente el Estado u otras entidades públicas y por las personas privadas, físicas o jurídicas, gestoras de servicios públicos en lo relacionado con la gestión de dichos servicios. Los documentos con una antigüedad superior a los cuarenta años generados, conservados o reunidos en el ejercicio de sus actividades por las entidades y asociaciones de carácter político, sindical o religioso y por las entidades, fundaciones y asociaciones culturales y educativas de carácter privado. Los documentos con una antigüedad superior a cien años generados, conservados o reunidos por cualesquiera otras entidades particulares o personas físicas.

La Administración podrá en estos dos últimos supuestos, declarar constitutivos del Patrimonio Documental bienes que, aun careciendo de esa antigüedad, merezcan tal calificación, de igual manera que puede excluir del mismo a los que, aun reuniendo las condiciones exigidas, se considere que no son dignos de pertenecer a él.

Por otro lado la Ley establece la obligación de llevar a cabo un Censo en el que deberían estar incluidos todos los documentos Archivísticos. Para realizarlo y puesto que hay Archivos de las Comunidades Autónomas, se elaboran convenios de colaboración con estas.

Scire. 11 : 1 (en.-jun. 2005) 175-186. ISSN 1135-3761. 


\section{Patrimonio documental en la universidad}

En la actualidad se está haciendo énfasis en la importancia que tiene la conservación del capital intelectual en las diferentes instituciones y en especial en aquellas donde se está en proceso continuo de generación de conocimiento. Por tanto, la universidad es una institución de educación en la cual se está en torno al consumo y a la generación de conocimientos nuevos, que con el paso del tiempo se convierten en verdaderos tesoros de riqueza intelectual. Sin embargo, son muy pocos los que tienen esta conciencia de la valoración del patrimonio documental en las universidades. Es posible que se tengan propuestas para la conservación de la memoria que está registrada en los archivos, pero el patrimonio documental de una institución no es exclusivamente la información que reposa en los archivos; también se constituyen en patrimonio documental los diferentes tipos de documentos en los que aparece registrada la producción académica, tales como libros, revistas, artículos de revistas, vídeos, CD-Roms, páginas web, folletos, afiches, obras de arte, composiciones musicales, entre otros.

La universidad, ente en el cual se aprecia el universo de manifestaciones académicas, culturales, sociales, lúdicas, políticas, es el indicador del estado de desarrollo de una sociedad y de una cultura. En la actualidad, con el fenómeno de la globalización y de la sociedad del conocimiento, la universidad entra en una nueva etapa de transformación, en la cual el conocimiento se constituye en el protagonista de su hacer cotidiano y de todas las actividades de docencia, investigación y extensión, motores del desempeño de toda entidad de educación superior. Al respecto, Guillermo Ruiz (2002, pp. 111-112), precisa sobre el panorama que atraviesa la universidad de hoy, frente a los retos que le plantea la sociedad del conocimiento, mediante la descripción de dos escenarios: el primer escenario se expresa de forma

optimista, donde la sociedad del conocimiento potenciaría el papel de la universidad como productora del conocimiento. Una sociedad atravesada por el conocimiento $-\mathrm{y}$ por sus consecuencias tecnológicas, sociales y educativas - y que estaría dominada por la preeminente institución del conocimiento: la universidad. Así, la sociedad dependería más aún de sus capacidades técnicas expertas; a la vez que la innovación y la tecnología dependerían aún más de la investigación científica, desarrollada principalmente en el seno de la universidad. El segundo escenario sería uno más negativo debido a que en la sociedad del conocimiento la universidad disputaría su lugar histórico con otras instituciones, las que emergerían debido a que en esta sociedad las instituciones tendrían que ser instituciones "inteligentes"; es decir, se trataría de organizaciones de aprendizaje y de investigación que competirían en un mercado de investigación y de conocimiento muy disputado (España, 2004).

Por tanto, la universidad debe estar atenta las nuevas perspectivas de desarrollo y evolución que le plantee la sociedad y además responder con soluciones 
adecuadas a la problemática que le presenta el mundo cambiante de hoy. Para ello, la universidad debe contar con un legado de saber, mediante el cual se estén aportando soluciones y opciones a los problemas que surjan continuamente, así como sentar las bases del conocimiento que se hubiera gestado durante el transcurso de su devenir histórico. Para ello, resulta de gran importancia la realización de una sistematización de toda su producción intelectual, desde el inicio de su actividad académica hasta la época actual. En consecuencia, se precisa de la construcción de un patrimonio documental que dé cuenta del aporte que le brinde la universidad a la comunidad durante el transcurrir de su trayectoria académica.

\section{Conformación del patrimonio documental en la universidad: caso específico de la Universidad de Antioquia (Colombia)}

En la actualidad, se está en el proceso de recopilar y valorar el patrimonio documental de la Universidad de Antioquia, entendido como toda aquella producción que se ha venido generando en ella. Por tanto se han fijado los siguientes lineamientos para adelantar la investigación sobre el patrimonio documental de esta Universidad en sus doscientos años de existencia.

\subsection{Objetivos}

El objetivo general es recuperar la memoria institucional de carácter bibliográfico, generada en las áreas administrativa, de Ciencias Exactas y Naturales, Ingeniería, Economía y Tecnología, Salud y Ciencias Sociales y representada en documentos publicados, coediciones, resultados de investigaciones, tesis de grado, materiales audiovisuales, obras de carácter artístico y demás publicaciones en diferentes soportes, producidos desde 1803 hasta 2002, como una contribución a la cultura y memoria de la institución, para facilitar el acceso en una forma ágil y eficiente a este patrimonio intelectual en estos campos del conocimiento.

Los objetivos específicos son los siguientes:

- Dar a conocer la diversidad de documentos que se han producido y el alcance de los mismos, mediante un inventario de la producción académica de las áreas administrativa, de Ciencias Exactas y Naturales, Ingeniería, Economía y Tecnología, Salud y Ciencias Sociales de la Universidad de Antioquia en sus doscientos años de existencia.

- Brindar a la comunidad nacional e internacional un estudio sistemático de la gran producción bibliográfica de las áreas antes mencionadas de la Universidad de Antioquia.

- Ofrecer un estudio valorativo (estado de arte) de carácter histórico de cada una de las áreas involucradas en la investigación. 
- Contribuir al desarrollo de la bibliografía nacional y brindar un aporte al control bibliográfico universal en diferentes campos del conocimiento.

\subsection{Metodología}

La investigación es de tipo histórico-bibliográfico descriptiva y la búsqueda en fuentes primarias y secundarias es fundamental. La consulta se realiza, en primer lugar, directamente en las dependencias que conforman las áreas objeto de estudio y luego en otras oficinas que puedan ser depositarias de la información.

Entre las actividades propuestas para la realización de la investigación se pueden resaltar la sensibilización al personal de las áreas objeto de la investigación, con el fin de lograr su comprensión y apoyo; rastrear y ubicar la información disponible sobre tesis y trabajos de grado, así como de las producciones académicas y administrativas de cada una de las áreas; recolectar, organizar, registrar y normalizar la información requerida, de acuerdo con estándares internacionales; evaluar la información recopilada, así como los registros que se elaboren de la misma, según la normatividad definida para su organización; sistematizar; ingresar la información en la base de datos seleccionada, de tal manera que se facilite su recuperación y su consulta; elaborar un documento electrónico e impreso que incluya la producción del conocimiento académico, investigador, administrativo y cultural de las dependencias del área; efectuar un estado de arte en el área de carácter histórico y valorativo sobre ciencia y tecnología en la Universidad de Antioquia; y, finalmente, diseñar y realizar actividades de socialización, conducentes al conocimiento, divulgación y promoción del trabajo, en eventos profesionales y revistas especializadas, entre otros medios.

\subsection{Fuentes y recursos de información}

La fuente de información por excelencia es la Universidad, en todas y cada una de sus dependencias administrativas, académicas, investigadoras y culturales, ya que se constituye en el recurso fundamental para el proceso de recolección, registro y almacenamiento de los documentos; así mismo, instituciones externas de carácter tanto local como internacional; $y$, finalmente, bases de datos de amplia cobertura. También se ha planeado realizar visitas académicas a diferentes instituciones culturales en Bogotá, tales como la Biblioteca Nacional, la Biblioteca Luis Ángel Arango, el Instituto Caro y Cuervo, las Academias de Medicina y de Ciencias Exactas, Físicas y Naturales, entre otras, donde puede rastrearse y recuperarse una cantidad significativa de la producción que compete a la investigación.

Los tipos de publicaciones a incluir son libros, monografías, folletos, revistas institucionales, artículos de revistas nacionales e internacionales, informes finales de investigación, manuales y módulos didácticos, publicaciones estudiantiles (revistas y periódicos), afiches y calendarios, tesis y trabajos de grado, 
publicados e inéditos, ponencias en eventos académicos y científicos, obras de carácter artístico, guiones y catálogos, patrimonio fílmico. Se aclara además que los documentos descritos anteriormente pueden estar en soporte manuscrito, impreso, audiovisual o electrónico (magnético, óptico).

En la realización de dicho repertorio bibliográfico, se excluyen documentos administrativos no publicados, tales como actas y circulares, así como materiales de archivo y prensa, las bases de datos y las páginas web. Igualmente se excluyen escritos sobre la Universidad de Antioquia, no solo por considerarse que no constituyen producción propia de la Universidad, sino porque su magnitud amerita una investigación diferente. En cuanto a publicaciones estudiantiles, no cubre las notas de clase y los productos de talleres y actividades académicas similares.

\subsection{Apoyo tecnológico}

En cuanto a las herramientas tecnológicas, es fundamental seleccionar un programa que permita diseñar una base de datos con los requerimientos propios de una bibliografía. Para ello se analizaron varios programas como CD-ISIS, Pro-Cite, Reference Manager y el OLIB entre otros, de los cuales se seleccionó el OLIB, debido a que resultaría más fácil diseñar una base de datos adjunta a la base de datos del sistema de bibliotecas de la Universidad de Antioquia y aprovechar la exportación de materiales que formarán parte del objeto de la investigación, y poder de esta forma estructurar un repertorio documental de una forma más ágil y rápida. Igualmente se utilizaron los recursos que provee la Internet, como los catálogos en línea de la Library of Congress, la Biblioteca Luis Ángel Arango y bibliotecas locales (Ruiz, 2002; Munera, 2003).

Para la realización de dicho repertorio documental, se definieron cuatro fases mediante las cuales se estructura el rastreo de las diferentes manifestaciones de conocimiento que se han producido en la Universidad. Estas fases son:

- Fase 1: Recopilación de toda la producción generada en las diferentes dependencias administrativas de la Universidad de Antioquia en sus doscientos años de existencia (1803-2003), así como la realización de un estado del arte, en el cual se pueda valorar dicha producción mediante el reconocimiento de tendencias que han marcado el accionar de la Universidad en diferentes épocas de su historia.

- Fase 2: Recopilación de lo producido en el área de Ciencias Exactas y Naturales, Ingeniería, Economía y Tecnología, y realización de un estado del arte, en el que se puedan identificar las diferentes corrientes y tendencias de tradición científica que se han desarrollado en estas áreas académicas de la Universidad, así como mirar el grado de aporte que han significado para el beneficio de la sociedad. 
- Fase 3: Recopilación de lo producido en el área de Ciencias de la Salud y estudio valorativo mediante la realización del estado del arte de dicha producción.

- Fase 4: Recopilación de la documentación producida en el área de Ciencias Sociales y Humanas, junto con el desarrollo de un estudio valorativo sobre el aporte de dichas áreas en beneficio de la sociedad y del crecimiento de las mismas.

En la actualidad se están desarrollando las dos primeras fases y además se están realizando como trabajos paralelos la sistematización de toda la nómina de empleados de la Universidad desde 1803 hasta nuestros días y el diseño de una base de datos "Patrimonio Documental", que se deriva del Sistema de Bibliotecas de la Universidad de Antioquia. A la base de datos del Patrimonio Documental se han importado del Catálogo del Sistema de Bibliotecas un total de 13.970 registros, cuyos datos se están revisando y corrigiendo. También se ingresará en ella la información de los documentos recopilados en las diferentes dependencias de la Universidad.

\section{Conclusiones}

El patrimonio como tal cumple una función fundamental en el proceso de reconocimiento no solo de la historia de los pueblos, sino también de la riqueza histórica, cultural, científica, natural, social y política de toda región y comunidad. En tal sentido, es necesario el fomento de su conservación y valoración por parte de las nuevas generaciones, las cuales, muy seguramente, tendrán en el patrimonio una base inmensa de conocimiento y sabiduría heredada de sus ancestros.

La universidad, en su rol de generadora de conocimiento para el beneficio de la sociedad, debe estar atenta a los retos que le impone el mundo globalizado de hoy; y debe, en igual medida, velar por que el patrimonio que construye dentro de su proceso de desarrollo sea eficientemente preservado y valorado por la comunidad a la que se proyecta. En tal sentido, es indispensable que se cree en las entidades de educación superior la conciencia de valorar y rescatar sus diversas manifestaciones de producción de conocimiento, ya que en ellas se refleja el grado de aporte que la universidad le ha ofrecido, le ofrece y le ofrecerá a la sociedad en general.

La importancia de recuperar el patrimonio documental universitario es inmensa, ya que permite visualizar el panorama de su labor en el transcurso del tiempo y la influencia que ha podido ejercer en ciertas etapas de su desarrollo en diferentes ámbitos. Lo cual se puede ilustrar con la experiencia que se está llevando a cabo en la Universidad de Antioquia de realizar la recopilación 
bibliográfica de todo el patrimonio documental de sus diferentes dependencias. Este tipo de trabajos puede dar lugar a la realización de estudios bibliométricos y cienciométricos sobre el grado de productividad de los diferentes estamentos universitarios, así como servir de base para el origen de nuevos proyectos de carácter científico.

\section{Referencias}

Galeano Londoño, José Ramiro (2003). Gestión del conocimiento en la universidad; la práctica universitaria. // Segunda Jornada de Gestión del Conocimiento. Medellín: Universidad de Antioquia, Escuela Interamericana de Bibliotecología, 2003.

España. Ministerio de Cultura. El patrimonio español. // Redes: Programa Patrimonio. es. Madrid: Ministerio de Industria, Turismo y Comercio, Fondo Europeo de Desarrollo Regional. URL: <http://www.mcu.es/patrimonio/jsp/plantilla. jsp? $\mathrm{id}=25 \&$ contenido $=/$ patrimonio/pp/nnp/patrimonioDocumental.html. Consultado: 2004-09-06.

Gobernación de Antioquia (1996). Secretaría de Educación y Cultura de Antioquia, Dirección de Extensión Cultural. Valoración del patrimonio cultural urbanístico. Medellín: La Secretaría, 1996. 5.

Gutiérrez Pantoja, Gabriel (1992). La investigación bibliográfica en el proceso de innovación tecnológica. // Boletín del Instituto de Investigaciones Bibliográficas. México. $2^{\mathrm{a}}$ época, 6 (1992) 141-190.

Hernández Hernández, Francisca. El patrimonio cultural: la memoria recuperada. Gijón: Trea, 2002. $462 \mathrm{p}$.

Lancaster, Frederic. Lineamientos para la recopilación de datos. México: UNAM, Centro de Investigaciones Bibliotecológicas, 1996. 12 p. (Folletos de apoyo profesional; 3).

Moreiro, José Antonio (1999). Introducción al estudio de la información y la documentación. Medellín: Universidad de Antioquia, 1999. 188 p.

Moreno Martínez, Luis (1998). Memoria bibliográfica institucional de la Universidad de Antioquia. Medellín: Escuela Interamericana de Bibliotecología, 1998. (Proyecto presentado al CODI en enero 30 de 1998).

Múnera Torres, María Teresa; Isaza Restrepo, Irma; Lotero Marín, Libia; Cadavid Arango, Carlos (2003). Proyecto de Investigación: Patrimonio Documental Universidad de Antioquia, 200 años. Medellín: U de A, 2003. 40 p.

Real Academia Española (2001). Diccionario de la lengua española. $22^{\mathrm{a}}$ ed. Madrid: Espasa, 2001.

República de Chile. Ministerio de Educación. Patrimonio Nacional: preservando la memoria, construyendo identidad. Chile: UNESCO: PIIE: MAV. (Recursos Educativos para Profesores). URL: <http://www.piie.cl/patrimonio/index.htm>. Consultado: 2004-09-06.

Ruiz, Guillermo (2002). La sociedad del conocimiento y la educación superior universitaria. // Revista Mexicana de Ciencias Políticas y Sociales. México. 45:185 (may-ago 2002) 111-112.

Scire. $11: 1$ (en.-jun. 2005) 175-186. ISSN 1135-3761. 
Santa, Eduardo (1973). El libro en Colombia. Bogotá: Instituto Colombiano de Cultura, 1973. 51-55.

UNESCO (1972). Conferencia General de la UNESCO en la $17^{a}$ Reunión, París, octubre 17 de 1972. URL: <http://whc.UNESCO.org/world_es.htm>. Consultado: 200403-04.

UNESCO. Patrimonio de la humanidad. http: //www.mcu.es/patrimonio/jsp/plantilla. jsp?id=36\&contenido=patrimonio/pei/ph(unesco.html.

Universidad de Antioquia (1998). Vicerrectoría de Investigaciones. Catálogo de investigaciones: grupos y centros de investigación 1998. Medellín: Universidad de Antioquia, 1998. $572 \mathrm{p}$. 